\title{
CAPABILITY OF NILPOTENT PRODUCTS OF CYCLIC GROUPS
}

\author{
ARTURO MAGIDIN \\ Department of Mathematics \\ University of Louisiana at Lafayette \\ 217 Maxim Doucet Hall \\ P.O. Box 41010 \\ magidin@member.ams.org
}

Published in J. Group Theory 8 (2005), pp. 431-452.

Errata added on 4/10/2006 - Updated on 5/6/2006

2000 Mathematics Subject Classification. Primary 20D15, Secondary 20 F12. 
Abstract. A group is called capable if it is a central factor group. We consider the capability of nilpotent products of cyclic groups, and obtain a generalization of a theorem of Baer for the small class case. The approach is also used to obtain some recent results on the capability of certain nilpotent groups of class 2 . We also prove a necessary condition for the capability of an arbitrary $p$-group of class $k$, and some further results.

\section{INTRODUCTION}

In his landmark paper on the classification of finite $p$-groups 11, P. Hall remarked that:

The question of what conditions a group $G$ must fulfil in order that it may be the central quotient group of another group $H$, $G \cong H / Z(H)$, is an interesting one. But while it is easy to write down a number of necessary conditions, it is not so easy to be sure that they are sufficient.

Following M. Hall and Senior [10, we make the following definition:

Definition 1.1. A group $G$ is said to be capable if and only if there exists a group $H$ such that $G \cong H / Z(H)$; equivalently, if and only if $G$ is isomorphic to the inner automorphism group of a group $H$.

Capability of groups was first studied by R. Baer in [3], where, as a corollary of some deeper investigations, he characterised the capable groups that are a direct sum of cyclic groups. Capability of groups has received renewed attention in recent years, thanks to results of Beyl, Felgner, and Schmid [5] characterising the capability of a group in terms of its epicenter; and more recently to work of Graham Ellis 6 ] that describes the epicenter in terms of the nonabelian tensor square of the group. The epicenter was used in [5] to characterize the capable extra-special $p$-groups; and the nonabelian tensor square was used in [1] to characterize the capable 2-generator finite $p$-groups of odd order and class 2 .

While the nonabelian tensor product has proven very useful in the study of capable groups, it does seem to present certain limitations. At the end of [1, for example, the authors note that their methods require "very explicit knowledge of the groups" in question.

In the present work, we will use "low-tech" methods to obtain a number of results on capability of finite $p$-groups. They rely only on commutator calculus, and so may be more susceptible to extension than results that require explicit knowledge of the nonabelian tensor square of a group. In particular, we will prove a generalization of Baer's Theorem characterising the capable direct sums of cyclic groups to the $k$-nilpotent products of cyclic $p$-groups with $k<p$, and with $k=p=2$.

One weakness in our results should be noted explicitly: Baer's Theorem fully characterizes the capable finitely generated abelian groups, because every finitely generated abelian group can be expressed as a direct sum of cyclic groups. Our generalization does not provide a result of similar reach, because whenever $k>1$ there exist finite $p$-groups of class $k$ that are not a $k$-nilpotent product of cyclic $p$-groups, even if we restrict to $p>k$. On the other hand, every finite $p$-group of class $k$ is a quotient of a $k$-nilpotent product of cyclic $p$-groups, so some progress can be made from this starting point. 
The paper is organized as follows: in Section 2 we present the basic definitions and notation. We proceed in Section 3 to establish a necessary condition for capability which extends an observation of P. Hall. In Section 4 we first describe the center of a $k$-nilpotent product of cyclic $p$-groups, where $p$ is a prime satisfying $p \geq k$, and then use this description to prove Theorem 4.5] the promised generalization of Baer's Theorem. In Section 5 we characterize the capable 2-nilpotent products of cyclic 2-groups. Then in Section [6 we use our results on capable 2nilpotent products of cyclic $p$-groups to derive a characterization of the capable 2 -generated nilpotent $p$-groups of class two, $p$ an odd prime (recently obtained through different methods by Bacon and Kappe), and some other related results, by way of illustration of how our methods may be used as a starting point for further investigations.

The main results are Theorem 3.19 giving a necessary condition for capability of a finite $p$ group of class $k$, and Theorems 4.5 and 5.2 characterising the capable $k$-nilpotent products of cyclic $p$-groups for $p>k$ and for $k=p=2$.

\section{Definitions and notation}

All maps will be assumed to be group homomorphisms. All groups will be written multiplicatively, unless we explicitly state otherwise.

Let $G$ be a group. The center of $G$ is denoted by $Z(G)$. The identity element of $G$ will be denoted by $e$.

Let $x \in G$. We say that $x$ is of exponent $n$ if and only if $x^{n}=e$; we say $x$ is of order $n>0$ if and only if $x^{n}=e$ and $x^{k} \neq e$ for all $k, 0<k<n$.

The commutator $[x, y]$ of two elements $x$ and $y$ is defined to be $[x, y]=x^{-1} y^{-1} x y$; given two subsets (not necessarily subgroups) $A$ and $B$ of $G$, we let $[A, B]$ be the subgroup generated by all elements of the form $[a, b]$, with $a \in A$ and $b \in B$.

The lower central series of $G$ is the sequence of subgroups defined by $G_{1}, G_{2}, \ldots$, where $G_{1}=G, G_{n+1}=\left[G_{n}, G\right]$. We say a group $G$ is nilpotent of class (at most) $n$ if and only if $G_{n+1}=\{e\}$; we will often drop the "at most" clause, it being understood. The nilpotent groups of class 1 are the abelian groups. Note that $G$ is nilpotent of class $n$ if and only if $G_{n} \subset Z(G)$. The class of all nilpotent groups of class at most $k$ is denoted by $\mathfrak{N}_{k}$; it is a variety of groups in the sense of General Algebra; we refer the reader to Hanna Neumann's excellent book [18.

We will write commutators left-normed, so that $\left[a_{1}, a_{2}, a_{3}\right]=\left[\left[a_{1}, a_{2}\right], a_{3}\right]$, etc.

The following properties of the lower central series are well-known. See for example 9]:

Definition 2.1. Let $g \in G, g \neq e$. We define $W(g)$ to be $W(g)=k$ if and only if $g \in G_{k}$ and $g \notin G_{k+1}$. We also set $W(e)=\infty$.

Proposition 2.2. Let $G$ be a group.

(i) For all $a, b \in G, W([a, b]) \geq W(a)+W(b)$.

(ii) If $W\left(a_{i}\right)=w_{1}$ and $W\left(b_{j}\right)=w_{2}$, then

$$
\left[\prod_{i=1}^{I} a_{i}^{\alpha_{i}}, \prod_{j=1}^{J} b_{j}^{\beta_{j}}\right] \equiv \prod_{i=1}^{I} \prod_{j=1}^{J}\left[a_{i}, b_{j}\right]^{\alpha_{i} \beta_{j}} \quad\left(\bmod G_{w_{1}+w_{2}+1}\right) .
$$

(iii) If $a \equiv c\left(\bmod G_{W(a)+1}\right)$ and $b \equiv d\left(\bmod G_{W(b)+1}\right)$, then

$$
[a, b] \equiv[c, d] \quad\left(\bmod G_{W(a)+W(b)+1}\right) .
$$


(iv) A variant of the Jacobi identity:

$$
[a, b, c][b, c, a][c, a, b] \equiv e \quad\left(\bmod G_{W(a)+W(b)+W(c)+1}\right) .
$$

The following identities may be verified by direct calculation:

$$
\begin{aligned}
{[x y, z] } & =[x, z][x, z, y][y, z] \\
{[x, y z] } & =[x, z][z,[y, x]][x, y] . \\
{\left[a^{r}, b^{s}\right] } & \equiv[a, b]^{r s}[a, b, a]^{s\left(\begin{array}{c}
r \\
2
\end{array}\right)}[a, b, b]^{r\left(\begin{array}{c}
s \\
2
\end{array}\right)} \quad\left(\bmod G_{4}\right) \\
{\left[b^{r}, a^{s}\right] } & \equiv[a, b]^{-r s}[a, b, a]^{-r\left(\begin{array}{c}
s \\
2
\end{array}\right)}[a, b, b]^{-s\left(\begin{array}{c}
r \\
2
\end{array}\right)} \quad\left(\bmod G_{4}\right),
\end{aligned}
$$

where $\left(\begin{array}{l}r \\ 2\end{array}\right)=\frac{r(r-1)}{2}$ for all integers $r$.

Nilpotent product of groups. The nilpotent products of groups were introduced by Golovin [8] as examples of regular products of groups. Although defined in a more general context in which there is no restriction on the groups involved, our definition will be restricted to the situation we are interested in.

Definition 2.7. Let $A_{1}, \ldots, A_{n} \in \mathfrak{N}_{k}$. The k-nilpotent product of $A_{1}, \ldots, A_{n}$, denoted by $A_{1} \amalg^{\mathfrak{N}_{k}} \cdots \amalg^{\mathfrak{N}_{k}} A_{n}$, is defined to be the group $G=F / F_{k+1}$, where $F$ is the free product of the $A_{i}, F=A_{1} * \cdots * A_{n}$, and $F_{k+1}$ is the $(k+1)$-st term of the lower central series of $F$.

Note that the "1-nilpotent product" is simply the direct sum. Also, if the $A_{i}$ are in $\mathfrak{N}_{k-1}$, and $G$ is the $k$-nilpotent product of the $A_{i}$, then the $(k-1)$-nilpotent product of the $A_{i}$ is isomorphic to $G / G_{k}$.

The use of the coproduct notation does not appear to be standard in the literature, but there is a good reason to use it: the $k$-nilpotent product as defined above is the coproduct (in the sense of category theory) in the category $\mathfrak{N}_{k}$.

Basic commutators. The collection process of M. Hall gives normal forms for relatively free groups in $\mathfrak{N}_{k}$, and in some other special cases. The concept of basic commutators is essential in this developement. The definition of basic commutators seems to vary in the literature (and sometimes even within the same work; cf. $§ 11.1$ and $\S 12.3$ in [9]). The definition we will use in the present work is that which appears in $\S 12.3$ of [9], where the ordering of commutators of weight $n>1$ is given by letting $\left[x_{1}, y_{1}\right]<\left[x_{2}, y_{2}\right]$ if and only if $y_{1}<y_{2}$ or $y_{1}=y_{2}$ and $x_{1}<x_{2}$ (lexicographically from right to left); here $\left[x_{1}, y_{1}\right]$ and $\left[x_{2}, y_{2}\right]$ are basic commutators of weight $n$.

For an exposition of the collection process, we direct the reader to Chapter 11 in 9. The main consequences are Hall's Basis Theorem [9] Theorem 11.2.4] and the collection formulas [9 \$12.3]. We will use a slightly more precise version of the collection formulas in Section 3 below. We will also use later on a special case of the generalization of the Basis Theorem due to R.R. Struik [19] Theorem 3]; it states that if we take the $k$-nilpotent product of $n$ cyclic $p$-groups, $p \geq k$, generated by $x_{1}, \ldots, x_{n}$, then there is a normal form for the elements as products $\prod c_{i}^{a_{i}}$, where $c_{1}<c_{2}<\cdots$ are the basic commutators on the $x_{i}$ of weight less than or equal to $k$, and the exponent $a_{i}$ is taken modulo the smallest of the orders of the generators that appear in the full expression of $c_{i}$.

A corollary of this result is that if $F$ is the $k$-nilpotent product of the cyclic $p$ groups, $p \geq k$, then $F_{i} / F_{i+1}$ is abelian with basis given by the basic commutators of weight exactly $i, i=1, \ldots, k$. 


\section{A NECESSARY CONDITION}

In this section we give a necessary condition for capability of finite $p$-groups based on the orders of the elements on a minimal generating set. In the case of small class (that is, when $G$ is a $p$-group with $G \in \mathfrak{N}_{k}$ and $p>k$ ), the condition reduces to an observation which goes back at least to P. Hall (penultimate paragraph in pp. 137 in 11). Although Hall only considers bases in the sense of his theory of regular $p$-groups, his argument is essentially the same as the one we present. However, Hall's result may not be very well known, since it is only mentioned in passing; see for example [1, Theorem 4.4].

We begin by recalling three consequences of the collection process:

Lemma 3.1 (Lemma H1 in [19]). Let $x, y$ be any elements of a group; let $c_{1}, c_{2}, \ldots$ be the sequence of basic commutators of weight at least two in $x$ and $[x, y]$, in ascending order. Then

$$
\left[x^{\alpha}, y\right]=[x, y]^{\alpha} c_{1}^{f_{1}(\alpha)} c_{2}^{f_{2}(\alpha)} \cdots c_{i}^{f_{i}(\alpha)} \cdots
$$

where

$$
f_{i}(\alpha)=a_{1}\left(\begin{array}{l}
\alpha \\
1
\end{array}\right)+a_{2}\left(\begin{array}{l}
\alpha \\
2
\end{array}\right)+\cdots+a_{w_{i}}\left(\begin{array}{c}
\alpha \\
w_{i}
\end{array}\right),
$$

where $a_{i} \in \mathbb{Z}$, and $w_{i}$ is the weight of $c_{i}$ in $x$ and $[x, y]$. If the group is nilpotent, then the expression in (3.2) gives an identity, and the sequence of commutators terminates.

Lemma 3.4 (Lemma H2 in [19]). Let $\alpha$ be a fixed integer, and $G$ a nilpotent group of class at most $k$. If $b_{j} \in G$ and $r<k$, then

$$
\left[b_{1}, \ldots, b_{i-1}, b_{i}^{\alpha}, b_{i+1}, \ldots, b_{r}\right]=\left[b_{1}, \ldots, b_{r}\right]^{\alpha} v_{1}^{f_{1}(\alpha)} v_{2}^{f_{2}(\alpha)} \cdots v_{t}^{f_{t}(\alpha)}
$$

where the $v_{k}$ are (not necessarily basic) commutators in $b_{1}, \ldots, b_{r}$ of weight strictly greater than $r$, and every $b_{j}, 1 \leq j \leq r$ appears in each commutator $v_{k}$. Furthermore, the $f_{i}$ are of the form (3.3), with $a_{j} \in \mathbb{Z}$, and $w_{i}=w_{i}^{\prime}-(r-1)$, where $w_{i}^{\prime}$ is the weight of $v_{i}$ in the $b_{i}$.

We find 3.5 useful in situations when we have commutators in some terms, some of which are shown as powers, and we want to "pull the exponent out." At other times, we want to reverse the process and pull the exponents "into" a commutator. In such situations, we use (3.5) to express $\left[b_{1}, \ldots, b_{r}\right]^{\alpha}$ in terms of other commutators. We will call the resulting identity (3.5); that is:

3.5) $\left[b_{1}, \ldots, b_{r}\right]^{\alpha}=\left[b_{1}, \ldots, b_{i-1}, b_{i}^{\alpha}, b_{i+1}, \ldots, b_{r}\right] v_{t}^{-f_{t}(\alpha)} \cdots v_{2}^{-f_{2}(\alpha)} v_{1}^{-f_{1}(\alpha)}$,

with the understanding that we will only do this in a nilpotent group so that the formula makes sense.

Lemma 3.6 (Theorem 12.3.1 in [9]). Let $x_{1}, \ldots, x_{s}$ be any s elements of a group. Let $c_{1}, c_{2}, \ldots$ be the basic commutators in $x_{1}, \ldots, x_{s}$ of weight at least 2 , written in increasing order. Then

$$
\left(x_{1} \cdots x_{s}\right)^{\alpha}=x_{1}^{\alpha} x_{2}^{\alpha} \cdots x_{s}^{\alpha} c_{1}^{f_{1}(\alpha)} \cdots c_{i}^{f_{i}(\alpha)} \cdots
$$

where $f_{i}(\alpha)$ is of the form (3.3), with $a_{j}$ integers that depend only on $c_{i}$ and not on $\alpha$, and $w_{i}$ is the weight of $c_{i}$ in the $x_{j}$. If the group is nilpotent, then equation (3.7) is satisfied as an identity in the group, and the sequence of commutators terminates. 
The following lemma is easily established by induction on the weight:

Lemma 3.8. Let $F(y, z)$ be the free group on two generators. Then every basic commutator of weight $\geq 3$ is of the form

$$
\left[z, y, y, c_{4}, \ldots, c_{r}\right] \quad \text { or } \quad\left[z, y, z, c_{4}, \ldots, c_{r}\right]
$$

where $r \geq 3$, and $c_{4}, \ldots, c_{r}$ are basic commutators in $y$ and $z$.

The main idea in our development is as follows: if we know that $\left[z, y^{p^{i}}\right]$ centralizes $\langle y, z\rangle$ in a group $G \in \mathfrak{N}_{k}$, for some prime $p$ and all integers $i$ greater than or equal to a given bound $a$, then we want to prove that a commutator of the form $\left[z^{p^{n}}, y\right]$ is equal to $\left[z, y^{p^{n}}\right]$. To accomplish this, we observe that a basic commutator of weight $k$ will have exponent $p^{a}$, since we may simply use Lemma 3.8]and Proposition 2.2 (ii) to pull the exponent into the second entry of the bracket. An arbitrary commutator of weight $k$ will also have the same exponent, since $G_{k}$ is abelian. For a basic commutator of weight $k-1$ we may use (3.5) and deduce that a sufficiently high power of $p$ will again yield the trivial element, by bounding below the power of $p$ that divides the exponents $f_{i}\left(p^{n}\right)$. Then we apply Lemma 3.6 to deal with an arbitrary element of $G_{k-1}$. Continuing in this way, we can show that $\langle y, z\rangle_{3}$ is of exponent $p^{M}$ for some $M$, and we will obtain the desired result by applying Lemma 3.1 to $\left[z^{p^{N}}, y\right]$ and $\left[z, y^{p^{N}}\right]$ for a suitably chosen large $N$, thus showing that they are both equal to $[z, y]^{p^{N}}$. Most of the work will go into obtaining a good estimate on how large this "large $N$ " has to be for everything to work.

If $p$ is a prime and $a$ is a positive integer, we let $[a]_{p}$ denote the exact $p$-divisor of $a$; that is, we say that $[a]_{p}=r$ if $p^{r} \mid a$ and $p^{r+1} \not\left\langle a\right.$. Formally, we set $[0]_{p}=\infty$. A classical theorem of Kummer implies that if $a$ is a positive integer, $0<a \leq p^{n}$, then

$$
\left[\left(\begin{array}{c}
p^{n} \\
a
\end{array}\right)\right]_{p}=n-[a]_{p} .
$$

Recall that if $x$ is a real number, then $\lfloor x\rfloor$ denotes the floor of $x$, the largest integer smaller than or equal to $x$.

Proposition 3.10. Let $p$ be a prime, $n$ a positive integer, and $m$ an integer with $0<m \leq p^{n}$. If $a_{1}, \ldots, a_{m}$ are integers, then

$$
a_{1}\left(\begin{array}{c}
p^{n} \\
1
\end{array}\right)+a_{2}\left(\begin{array}{c}
p^{n} \\
2
\end{array}\right)+\cdots+a_{m}\left(\begin{array}{c}
p^{n} \\
m
\end{array}\right) \equiv 0 \quad\left(\bmod p^{n-d}\right),
$$

where $d$ is the smallest integer such that $p^{d+1}>m$; that is, $d=\left\lfloor\log _{p}(m)\right\rfloor$.

Proof. Write $m=m_{0}+m_{1} p+\cdots+m_{d} p^{d}$, with $0 \leq m_{i}<p$ and $m_{d}>0$. Then for all integers $k$ between 1 and $m, 0 \leq[k]_{p} \leq d$. Therefore, $n-[k]_{p} \geq n-d$, so each summand in (3.11) is divisible by $p^{n-d}$, as claimed.

Lemma 3.12. Let $k \geq 3$, and let $G \in \mathfrak{N}_{k}$. Let $p$ be a prime, $a \geq 0$ an integer, and $y, z \in G$. Suppose that

$$
\forall i \geq a, \quad\left[z, y^{p^{i}}, y\right]=\left[z, y^{p^{i}}, z\right]=e .
$$

Then $\langle y, z\rangle_{k}$ is of exponent $p^{a}$, and $\langle y, z\rangle_{k-1}$ is of exponent $p^{a+\left\lfloor\frac{1}{p-1}\right\rfloor}$. 
Proof. It follows by (3.5), (3.9), and our assumption that

$$
\left[z, y, w, c_{4}, \ldots\right]^{p^{a}}=\left[z, y^{p^{a}}, w, c_{4}, \ldots\right]=e
$$

where $w \in\{y, z\}$, and $\left[z, y, w, c_{4}, \ldots\right]$ is a basic commutator of weight $k$. Since $\langle y, z\rangle_{k}$ is abelian and generated by the basic commutators, this proves the first part of the statement.

Now let $c \in\langle y, z\rangle_{k-1}$ be a basic commutator of weight exactly $k-1$, and let $N \geq a$. Expressing $c$ in the form given in (3.9), say $c=\left[z, y, w, c_{r}, \ldots\right]$ with $w \in\{y, z\}$, then (3.5) and our assumption yield

$$
c^{p^{N}}=\left[z, y, w, c_{4}, \ldots\right]^{p^{N}}=v_{t}^{-f_{t}\left(p^{N}\right)} \cdots v_{1}^{-f_{1}\left(p^{N}\right)},
$$

where all the $v_{i}$ are of weight $k$. The corresponding exponents are of the form

$$
f_{i}\left(p^{N}\right)=a_{1}\left(\begin{array}{c}
p^{N} \\
1
\end{array}\right)+a_{2}\left(\begin{array}{c}
p^{N} \\
2
\end{array}\right)
$$

By Proposition 3.10 this expression is divisible by $p^{N-\left\lfloor\log _{p}(2)\right\rfloor}$, and so whenever $N \geq a+\left\lfloor\log _{p}(2)\right\rfloor$, we have $c^{p^{N}}=e$. Therefore, every basic commutator of weight exactly $k-1$ is of exponent $p^{a+\left\lfloor\log _{p}(2)\right\rfloor}$. Since $k>2, G_{k-1}$ is abelian as well; as every generator is of exponent $p^{a+\left\lfloor\log _{p}(2)\right\rfloor}$, the theorem is now proven by noting that $\left\lfloor\log _{p}(2)\right\rfloor=\left\lfloor\frac{1}{p-1}\right\rfloor$.

We will need the following technical lemma:

Lemma 3.14. Let $k$ and $n$ be positive integers, with $n>1$. Then

$$
\max _{1 \leq s \leq k}\left(\left\lfloor\frac{k-s}{n-1}\right\rfloor+\left\lfloor\log _{n}(s+1)\right\rfloor\right)=\left\lfloor\frac{k}{n-1}\right\rfloor .
$$

If $k \geq n-1$ then the maximum is always attained at $s=n-1$.

Proof. Fix $k$ and $n$; we may assume $k \geq n-1$. Let

$$
f(s)=\frac{k-s}{n-1}+\left\lfloor\log _{n}(s+1)\right\rfloor, \quad s \in[1, k] .
$$

The function $f(s)$ is piecewise strictly decreasing on $[1, k]$, and continuous from the right at every point. So the maximum of $f(s)$ on $[1, k]$ will be achieved at $s=1$, or at a point where $f(s)$ is not continuous. The points of discontinuity are the points of the form $s=n^{r}-1$ where $1 \leq r \leq\left\lfloor\log _{n}(k+1)\right\rfloor$. At $s=1$, we have

$$
f(1)=\frac{k-1}{n-1}+\left\lfloor\log _{n}(2)\right\rfloor \leq \frac{k}{n-1},
$$

with equality if and only if $n=2$. At the points of discontinuity we have

$$
\begin{aligned}
f\left(n^{r}-1\right) & =\frac{k}{n-1}-\frac{n^{r}-1}{n-1}+r \\
& =\frac{k}{n-1}-\left(1+n+n^{2}+\cdots+n^{r-1}\right)+r \leq \frac{k}{n-1},
\end{aligned}
$$

with equality if and only if $r=1$. Thus the maximum value of $f(s)$ is equal to $\frac{k}{n-1}$, always achieved at $s_{0}=n-1$. The result now follows by taking $\lfloor f(s)\rfloor$. 
Lemma 3.15 (cf. Lemma 8.83 in [16]). Let $G$ be a group, with $G \in \mathfrak{N}_{k}$ and $k \geq 3$. Furthermore, let $p$ be a prime, $a>0$ an integer, and $y, z \in G$. Assume that $a, y, z$, and $G$ satisfy (3.13). Then $\langle y, z\rangle_{k-m}$ is of exponent $p^{a+\left\lfloor\frac{m}{p-1}\right\rfloor}$ for $m=0,1, \ldots,(k-3)$.

Proof. We proceed by induction on $m$. Lemma 3.12 proves cases $m=0,1$, so we let $m \geq 2$.

Assume the result is true for $j=0,1, \ldots, m-1$. First, we consider a basic commutator $c$ of weight exactly $k-m$. We may express $c$ as in (3.9), and applying (3.5) to $c^{p^{N}}$ we obtain

$$
c^{p^{N}}=\left[z, y^{p^{N}}, w, c_{4}, \ldots, c_{r}\right] v_{t}^{-f_{t}\left(p^{N}\right)} \cdots v_{2}^{-f_{2}\left(p^{N}\right)} v_{1}^{-f_{1}\left(p^{N}\right)},
$$

where $w \in\{y, z\}, c_{4}, \ldots, c_{r}$ are basic commutators in $z$ and $y$, and the $v_{i}$ are commutators in $z, y, w, c_{4}, \ldots, c_{r}$; we know that each of $z, y, w, c_{4}, \ldots, c_{r}$ appears at least once in each $v_{i}$, and that we may express the weight of $v_{i}$ on $z, y, w, c_{4}, \ldots, c_{r}$ as $r+s$ for some positive integer $s$. Thus, we may conclude that if the weight of $v_{i}$ in $z, y, w, c_{4}, \ldots, c_{r}$ is $r+s$, then $W\left(v_{i}\right) \geq(k-m)+s$. In particular, we consider only those commutators with $1 \leq s \leq m$.

If $v_{i}$ is of weight $r+s$ in $z, y, w, c_{4}, \ldots, c_{r}$, then by Lemma 3.4 we have that

$$
f_{i}\left(p^{N}\right)=a_{1}\left(\begin{array}{c}
p^{N} \\
1
\end{array}\right)+\cdots+a_{s+1}\left(\begin{array}{c}
p^{N} \\
s+1
\end{array}\right) .
$$

Therefore, $f_{i}\left(p^{N}\right)$ is divisible by $p^{N-\left\lfloor\log _{p}(s+1)\right\rfloor}$. Since $v_{i} \in\langle y, z\rangle_{(k-(m-s))}$, by the induction hypothesis we know that $v_{i}^{-f_{i}\left(p^{N}\right)}$ is trivial whenever $N-\left\lfloor\log _{p}(s+1)\right\rfloor \geq$ $a+\left\lfloor\frac{m-s}{p-1}\right\rfloor$. Therefore, if

$$
N \geq a+\left\lfloor\frac{m-s}{p-1}\right\rfloor+\left\lfloor\log _{p}(s+1)\right\rfloor
$$

for $s=1 \ldots, m$, then we may conclude that $c^{p^{N}}=e$. By Lemma 3.14 the largest of these values is $a+\left\lfloor\frac{m}{p-1}\right\rfloor$, which shows that the basic commutators of weight exactly $m-k$ are of exponent $p^{a+\left\lfloor\frac{m}{p-1}\right\rfloor}$, as desired.

Now take an arbitrary element $c$ of $\langle y, z\rangle_{(k-m)}$, and write $c=d_{1} \cdots d_{r}$, where $d_{i}=c_{i}^{\beta_{i}}$ is the power of a basic commutator of weight at least $k-m$ in $y$ and $z$, and $c_{1}<\cdots<c_{r}$. To calculate $c^{p^{N}}$, we apply Lemma 3.6 to this expression. We obtain:

$$
\left(d_{1} \cdots d_{r}\right)^{p^{N}}=c_{1}^{\beta_{1} p^{N}} c_{2}^{\beta_{2} p^{N}} \cdots c_{r}^{\beta_{r} p^{N}} u_{1}^{f_{1}\left(p^{N}\right)} \cdots u_{i}^{f_{i}\left(p^{N}\right)} \cdots
$$

where $f_{i}\left(p^{N}\right)$ is of the form (3.3), with $w_{i}$ the weight of $u_{i}$ in the $d_{j}$. If we let the weight of $u_{i}$ in the $d_{j}$ be equal to $s$, then we know that $2 \leq s$. Since $W\left(d_{i}\right) \geq(m-k)$, we have that $W\left(u_{i}\right) \geq s(m-k)$. In particular, we may restrict our attention to values of $s$ satisfying $2 \leq s \leq\left\lfloor\frac{k}{k-m}\right\rfloor$.

If $u_{i}$ is of weight $s$ in the $d_{j}$, then it lies in $\langle y, z\rangle_{k-(m-(s-1)(k-m))}$, so by the induction hypothesis it is of exponent $p^{a+\left\lfloor\frac{m-(s-1)(k-m)}{p-1}\right\rfloor}$. By (3.3) and Proposition 3.10 we have that $f_{i}\left(p^{N}\right)$ is a multiple of $p^{N-\left\lfloor\log _{p}(s)\right\rfloor}$; thus we can guarantee that $u_{i}^{f_{i}\left(p^{N}\right)}$ is trivial if

$$
N \geq a+\left\lfloor\frac{m-(s-1)(k-m)}{p-1}\right\rfloor+\left\lfloor\log _{p}(s)\right\rfloor .
$$


Since $s \geq 2$ and $k-m \geq 3$, it is clear that this value will certainly be no larger than $a+\left\lfloor\frac{m}{p-1}\right\rfloor$ by Lemma 3.14 so we conclude that any element of $\langle y, z\rangle_{(k-m)}$ is of exponent $p^{a+\left\lfloor\frac{m}{p-1}\right\rfloor}$, as claimed.

Theorem 3.16 (cf. Corollary 8.84 in [16]). Let $k \geq 1$ and $G \in \mathfrak{N}_{k+1}$. Furthermore, let $p$ be a prime, $a>0$ an integer, and $y$ and $z$ elements of $G$. Assume that

$$
\forall i \geq a, \quad\left[z, y^{p^{i}}, y\right]=\left[z, y^{p^{i}}, z\right]=e .
$$

If $N \geq a+\left\lfloor\frac{k-1}{p-1}\right\rfloor$, then $\left[z^{p^{N}}, y\right]=[z, y]^{p^{N}}=\left[z, y^{p^{N}}\right]$.

Proof. Applying Lemma 3.1 to $\left[z^{p^{N}}, y\right]$ yields:

$$
\left[z^{p^{N}}, y\right]=[z, y]^{p^{N}} v_{1}^{f_{1}\left(p^{N}\right)} v_{2}^{f_{2}\left(p^{N}\right)} \cdots v_{t}^{f_{t}\left(p^{N}\right)},
$$

where $v_{1}, v_{2}, \ldots$ are the basic commutators of weight at least 2 in $z$ and $[z, y]$. If $v_{i}$ is of weight $s \geq 2$ in $z$ and $[z, y]$, then

$$
f_{i}\left(p^{N}\right)=a_{1}\left(\begin{array}{c}
p^{N} \\
1
\end{array}\right)+a_{2}\left(\begin{array}{c}
p^{N} \\
2
\end{array}\right)+\cdots+a_{s}\left(\begin{array}{c}
p^{N} \\
s
\end{array}\right),
$$

and we know that $v_{i} \in\langle y, z\rangle_{s+1}$. By Theorem $3.15 v_{i}^{f_{i}\left(p^{N}\right)}$ will be trivial if

$$
N \geq a+\left\lfloor\frac{(k+1)-(s+1)}{p-1}\right\rfloor+\left\lfloor\log _{p}(s)\right\rfloor
$$

This must hold for $s=2, \ldots, k$. We set $s^{\prime}=s-1$ and rewrite the above as:

$$
N \geq a+\left\lfloor\frac{(k-1)-s^{\prime}}{p-1}\right\rfloor+\left\lfloor\log _{p}\left(s^{\prime}+1\right)\right\rfloor,
$$

with $s^{\prime}=1, \ldots, k-1$. By Lemma 3.14 the largest value the right hand side takes is $a+\left\lfloor\frac{k-1}{p-1}\right\rfloor$. This proves that $\left[z^{p^{N}}, y\right]=[z, y]^{p^{N}}$ if $N \geq a+\left\lfloor\frac{k-1}{p-1}\right\rfloor$. The proof that $\left[z, y^{p^{N}}\right]=[z, y]^{p^{N}}$ is essentially the same.

Remark 3.17. The results above also hold if the identity in 3.13) is replaced by an identity in which $p^{i}$ is placed on any of the three entries in $[z, y, z]$, and in any of the three entries in $[z, y, y]$; for instance,

$$
\left[z^{p^{i}}, y, z\right]=\left[z, y, y^{p^{i}}\right]=e, \quad \text { or } \quad\left[z, y^{p^{i}}, z\right]=\left[z^{p^{i}}, y, y\right]=e,
$$

etc.

Lemma 3.18. Let $k \geq 1$ be a positive integer, $p$ a prime, and let $H$ be a nilpotent p-group of class $k+1$. Suppose that $y_{1}, \ldots, y_{r}$ are elements of $H$ such that their images generate $H / Z(H)$; assume further that the order of $y_{i} Z(H)$ in $H / Z(H)$ is $p^{\alpha_{i}}$, with $1 \leq \alpha_{1} \leq \cdots \leq \alpha_{r}$. Then $\alpha_{r} \leq \alpha_{r-1}+\left\lfloor\frac{k-1}{p-1}\right\rfloor$; that is,

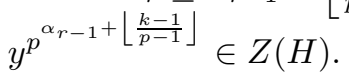

Proof. Since $H$ is generated by $y_{1}, \ldots, y_{r}$ and central elements, it is sufficient to prove that $\left[y_{r}^{p^{\alpha_{r-1}+\left\lfloor\frac{k-1}{p-1}\right\rfloor}}, y_{j}\right]=e$ for $j=1, \ldots, r-1$.

Note that $y_{j}^{p^{\alpha_{r-1}}}$ is central for $j=1, \ldots, r-1$, so that

$$
\forall i \geq \alpha_{r-1}, \quad\left[y_{r}, y_{j}^{p^{i}}, y_{j}\right]=\left[y_{r}, y_{j}^{p^{i}}, y_{r}\right]=e .
$$


Thus, we may apply Theorem 3.16 to conclude that

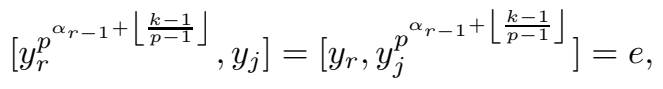

proving our lemma.

The necessary condition is now immediate:

Theorem 3.19 (P. Hall if $k<p$ [1]). Let $G$ be a nilpotent p-group of class $k$, with $k \geq 1$ and $p$ a prime. Furthermore, let $\left\{x_{1}, \ldots, x_{r}\right\}$ be a generating set for $G$ with $x_{i}$ of order $p^{\alpha_{i}}$, where $\alpha_{1} \leq \alpha_{2} \leq \cdots \leq \alpha_{r}$. If $G$ is capable, then $r>1$ and

$$
\alpha_{r} \leq \alpha_{r-1}+\left\lfloor\frac{k-1}{p-1}\right\rfloor \text {. }
$$

Proof. Since a center-by-cyclic group is abelian, the necessity of $r>1$ is clear. So assume that $G$ is capable, and $r>1$. Let $H$ be a $p$-group of class $k+1$ such that $G \cong H / Z(H)$. Let $y_{1}, \ldots, y_{r}$ be elements of $H$ that project onto $x_{1}, \ldots, x_{r}$, respectively. Then Lemma 3.18 gives the condition on $\alpha_{r}$, proving the theorem.

Remark 3.20. If $G$ is of small class then $\left\lfloor\frac{k-1}{p-1}\right\rfloor=0$, so Theorem 3.19 says that $\alpha_{r} \leq \alpha_{r-1}$; therefore, when $k<p$ the necessary condition becomes " $r>1$ and $\alpha_{r}=\alpha_{r-1} . "$ This is Hall's observation in 11], applied to a minimal generating set.

The obvious question to ask is whether the inequality is tight. It is clearly best possible if $k<p$. An easy case to consider for $k \geq p$ is $p=2$. In this case, the dihedral group of order $2^{k+1}$ is of class $k$, minimally generated by an element of order 2 and an element of order $2^{k}$; and its central quotient is isomorphic to the dihedral group of order $2^{k}$. Thus, the inequality is tight for all $k$ when $p=2$. The case of $k \geq p>2$ is more difficult, but once again there is a 2-generator capable group where the equality holds, with one generator of order $p$. This is shown in [17. These considerations lead to the following proposition.

Proposition 3.21 (17). For every $k \geq 1$ and every prime $p$ there exists a capable p-group of class $k$, minimally generated by an element of order $p$ and an element of order $p^{1+\left\lfloor\frac{k-1}{p-1}\right\rfloor}$. In particular, the bound in Theorem 3.19 is best possible.

\section{4. $k$-NILPOTENT PRODUCTS OF CYCLIC $p$-GROUPS, $k<p$}

In this section we describe the center of a $k$-nilpotent product of cyclic $p$-groups in the case $k \leq p$; as a result of this description, we will prove the promised generalization of Baer's Theorem for the small class case. The description is proven by induction on $k$, and we will need to do the case $k=2$ explicitly.

Lemma 4.1. Let $p$ be a prime and $C_{1}, \ldots, C_{r}$ be cyclic p-groups generated by $x_{1}, \ldots, x_{r}$, respectively, with $p^{\alpha_{i}}$ the order of $x_{i}$ and $1 \leq \alpha_{1} \leq \cdots \leq \alpha_{r}$. If $G=$ $C_{1} \amalg^{\mathfrak{N}_{2}} \ldots \amalg^{\mathfrak{N}_{2}} C_{r}$, then

$$
Z(G)=\left\langle x_{r}^{p^{\alpha_{r-1}}}, G_{2}\right\rangle .
$$

Proof. A theorem of T. MacHenry [14] shows that the cartesian [B, $A]$ of $A \amalg^{\mathfrak{N}_{2}} B$, with $A, B \in \mathfrak{N}_{2}$, is isomorphic to $B^{\mathrm{ab}} \otimes A^{\mathrm{ab}}$, the isomorphism being the one that sends $[b, a]$ to $\bar{b} \otimes \bar{a}$. Using the universal property of the coproduct, we map $G$ 
to $C_{i} \amalg^{\mathfrak{N}_{2}} C_{r}$ to conclude that $C_{i} \cap Z(G)=\{e\}$ for $i=1, \ldots, r-1$. Looking at $C_{r-1} \amalg^{\mathfrak{N}_{2}} C_{r}$ we also obtain that $C_{r} \cap Z(G)=\left\langle x_{r}^{p^{\alpha_{r}-1}}\right\rangle$, proving our claim.

We will also need two observations on basic commutators:

Lemma 4.2. Let $F$ be the free group on $x_{1}, \ldots, x_{r}$. Let $[u, v]$ be a basic commutator in $x_{1}, \ldots, x_{r}$, and assume that $\mathrm{wt}([u, v])=k \geq 2$.

(i) If $v \leq x_{r}$, then $\left[u, v, x_{r}\right]$ is a basic commutator in $x_{1}, \ldots, x_{r}$.

(ii) If $v>x_{r}$, then $\left[u, v, x_{r}\right] \equiv\left[v, x_{r}, u\right]^{-1}\left[u, x_{r}, v\right]\left(\bmod F_{k+2}\right)$. In addition, both $\left[v, x_{r}, u\right]$ and $\left[u, x_{r}, v\right]$ are basic commutators in $x_{1}, \ldots, x_{r}$.

Proof. Clause (i) follows from the definition of basic commutator, as does the claim in clause (ii) that $\left[v, x_{r}, u\right]$ and $\left[u, x_{r}, v\right]$ are basic commutators. The congruence in (ii) follows from Proposition 2.2 (iv).

Lemma 4.3. Let $F$ be the free group on $x_{1}, \ldots, x_{r}$, and let $k \geq 2$. Let $c_{1}, \ldots, c_{s}$ be the basic commutators in $x_{1}, \ldots, x_{r}$ of weight exactly $k$ listed in ascending order, and write $c_{i}=\left[u_{i}, v_{i}\right]$. Let $\beta_{1}, \ldots, \beta_{s}$ be any integers, and let $g=c_{1}^{\beta_{1}} \cdots c_{s}^{\beta_{s}}$. For $i=1, \ldots, s$, let $d_{i}$ and $f_{i}$ be defined by:

$$
\begin{aligned}
& d_{i}=\left\{\begin{array}{cc}
{\left[u_{i}, v_{i}, x_{r}\right]} & \text { if } v_{i} \leq x_{r} \\
{\left[v_{i}, x_{r}, u_{i}\right]^{-1}} & \text { if } v_{i}>x_{r} .
\end{array}\right. \\
& f_{i}=\left\{\begin{array}{cl}
e & \text { if } v_{i} \leq x_{r} \\
{\left[u_{i}, x_{r}, v_{i}\right]} & \text { if } v_{i}>x_{r} .
\end{array}\right.
\end{aligned}
$$

Then

$$
\left[g, x_{r}\right] \equiv \prod_{i=1}^{s} d_{i}^{\beta_{i}} f_{i}^{\beta_{i}} \quad\left(\bmod F_{k+2}\right)
$$

and, except for removing the trivial terms with $f_{i}=e$, this expression is in normal form for the abelian group $F_{k+1} / F_{k+2}$.

Proof. It is easy to verify that if $\left[u_{i}, v_{i}\right] \neq\left[u_{j}, v_{j}\right]$, then $d_{i}, f_{i}, d_{j}, f_{j}$ will be pairwise distinct, except perhaps in the case where $f_{i}=f_{j}=e$. Thus in the expression given for $\left[g, x_{r}\right]$, all terms are either powers of the identity or of pairwise distinct basic commutators. That the expression is indeed equal to $\left[g, x_{r}\right]$ follows from Proposition 2.2(ii).

We are now ready to prove our result:

Theorem 4.4. For a positive integer $k$ and a prime $p$ with $p \geq k$, let $C_{1}, \ldots, C_{r}$ be cyclic p-groups generated by $x_{1}, \ldots, x_{r}$ respectively, with $p^{\alpha_{i}}$ being the order of $x_{i}$, and assume that $1 \leq \alpha_{1} \leq \alpha_{2} \leq \cdots \leq \alpha_{r}$. If $G$ is the $k$-nilpotent product of the $C_{i}$, $G=C_{1} \amalg^{\mathfrak{N}_{k}} \cdots \amalg^{\mathfrak{N}_{k}} C_{r}$, then $Z(G)=\left\langle x_{r}^{p^{\alpha_{r}-1}}, G_{k}\right\rangle$.

Proof. That the center contains the right hand side follows from Lemma 3.18 and the properties of a $k$-nilpotent product. To prove the other inclusion, we proceed by induction on $k$. The result is trivially true for $k=1$, and Lemma 4.1 gives the result for $k=2$.

Assume the result is true for the $(k-1)$-nilpotent product of the $C_{i}, 2<k \leq p$, and let $K=C_{1} \amalg^{\mathfrak{N}_{k-1}} \cdots \amalg^{\mathfrak{N}_{k-1}} C_{r}$; that is, $K=G / G_{k}$.

By the induction hypothesis, we know that $Z(K)=\left\langle x_{r}^{p^{\alpha}{ }^{2-1}}, K_{k-1}\right\rangle$, and the center of $G$ is contained in the pullback of this subgroup. So we have the inclusions $\left\langle x_{r}^{p^{\alpha_{r-1}}}, G_{k}\right\rangle \subseteq Z(G) \subseteq\left\langle x_{r}^{p^{\alpha_{r-1}}}, G_{k-1}\right\rangle$. Let $g \in Z(G)$; multiplying by adequate 
elements of $G_{k}$ and an adequate power of $x_{r}^{p^{\alpha_{r-1}}}$, we may assume that $g$ is an element of $G_{k-1}$ which can be written in normal form as:

$$
g=\prod_{i=1}^{n} c_{i}^{\beta_{i}}
$$

where $c_{1}, \ldots, c_{n}$ are the basic commutators of weight exactly $k-1$ in $x_{1}, \ldots, x_{r}$, and the $\beta_{i}$ are integers on the relevant interval. If we prove that $g=e$, we will obtain our result.

Since $g \in Z(G)$, its commutator with $x_{r}$ is trivial. From Proposition 2.2 (ii) we have:

$$
e=\left[g, x_{r}\right]=\left[\prod_{i=1}^{n} c_{i}^{\beta_{i}}, x_{r}\right]=\prod_{i=1}^{n}\left[c_{i}, x_{r}\right]^{\beta_{i}} .
$$

For each $i=1, \ldots, n$, write $c_{i}=\left[u_{i}, v_{i}\right]$, with $u_{i}, v_{i}$ basic commutators. Let $d_{i}$ and $f_{i}$ be as in the statement of Lemma 4.3. Then we have

$$
e=\left[g, x_{r}\right]=\prod_{i=\ell}^{n} d_{i}^{\beta_{i}} f_{i}^{\beta_{i}} .
$$

Except for some $f_{i}$ which are trivial, the precise ordering of the remaining terms, and the exponents for the $d_{i}$ corresponding to $v_{i}>x_{r}$, this is already in normal form. The ordering of the nontrivial basic commutators is immaterial, since the $d_{i}$ and $f_{j}$ commute pairwise; and for those $d_{i}$ corresponding to $v_{i}>x_{r}$, we simply add the corresponding power of $p$ to the exponent $-\beta_{i}$ to obtain an exponent in the correct range (see [19, Theorem 3]). The expression is then in normal form, so the only way in which this product can be the trivial element is if $\beta_{i}=0$ for $i=1, \ldots, n$, proving that $g=e$, and so the theorem.

We now have the desired generalization:

Theorem 4.5 (Baer [3] for $k=1$ ). For $k$ a positive integer and $p$ a prime with $p>k$, let $C_{1}, \ldots, C_{r}$ be cyclic p-groups generated by $x_{1}, \ldots, x_{r}$ respectively, where $p^{\alpha_{i}}$ is the order of $x_{i}$ with $1 \leq \alpha_{1} \leq \alpha_{2} \leq \cdots \leq \alpha_{r}$. If $G$ is the $k$-nilpotent product of the $C_{i}, G=C_{1} \amalg^{\mathfrak{N}_{k}} \ldots \amalg^{\mathfrak{N}_{k}} C_{r}$, then $G$ is capable if and only if $r>1$ and $\alpha_{r-1}=\alpha_{r}$.

Proof. Necessity follows from Theorem 3.19. For sufficieny, let

$$
K=C_{1} \amalg^{\mathfrak{N}_{k+1}} \ldots \amalg^{\mathfrak{N}_{k+1}} C_{r} .
$$

Since $\alpha_{r-1}=\alpha_{r}$, we have from Theorem 4.4 that $Z(K)=K_{k+1}$, so $K / Z(K)=$ $K / K_{k+1} \cong G$, as desired.

Remark 4.6. The original statement of Baer's Theorem is not restricted to torsion groups, or even to finitely generated groups. The original result is:

Baer's Theorem (Corollary to Existence Theorem in [3]). A direct sum G of cyclic groups (written additively) is capable if and only if it satisfies the following two conditions:

(i) If the rank of $G / G_{\text {tor }}$ is 1 , then the orders of the elements in $G_{\text {tor }}$ are not bounded; and

(ii) If $G=G_{\text {tor }}$, and the rank of $\left(p^{i-1} G\right)_{p} /\left(p^{i} G\right)_{p}$ is 1 , then $G$ contains elements of order $p^{i+1}$, for all primes $p$; 
where $k G=\{k x \mid x \in G\}$, and $H_{p}=\{h \in H \mid$ ph $=0\}$ for any subgroup $H$ of $G$.

The reader may find it interesting to give a proof of Baer's Theorem using our methods and the 2-nilpotent product; a complete description of the multiplication table for the 2-nilpotent product is given by Golovin in 77. The proof of Baer's Theorem is then straightforward, and only complicated by the notation needed to consider infinite direct sums.

\section{The CASE $k=p=2$}

In this section we consider the smallest case that is not covered by our investigations so far: $k=p=2$. In this instance, Theorem 3.19 gives the condition $\alpha_{r} \leq \alpha_{r-1}+1$. We will prove that the condition is also sufficient for the case of the 2-nilpotent product of cyclic 2-groups.

As before, we start by examining the center of a 3-nilpotent product of cyclic 2-groups. Such a product was considered in detail by R.R. Struik in [19,20. To obtain uniqueness in the normal form, we must replace the basic commutators $[z, y, z]$ and $[z, y, y]$ with commutators $\left[z^{2}, y\right]$ and $\left[z, y^{2}\right]$, respectively, and adjust the ranges of the exponents accordingly. The normal form result we obtain with these changes is described in [19. Theorem 4]; explicitly, it states that if $C_{1}, \ldots, C_{r}$ are cyclic groups generated by $x_{1}, \ldots, x_{r}$ respectively, and if the order of $x_{i}$ is $2^{\alpha_{i}}$, $1 \leq \alpha_{1} \leq \cdots \leq \alpha_{r}$, then every element of the 3-nilpotent product of the $C_{r}$ may be written uniquely in normal form as:

$g=x_{1}^{\gamma_{1}} \cdots x_{r}^{\gamma_{r}} \prod_{1 \leq i<j \leq r}\left[x_{j}, x_{i}\right]^{\gamma_{j i}}\left[x_{j}^{2}, x_{i}\right]^{\gamma_{j i j}}\left[x_{j}, x_{i}^{2}\right]^{\gamma_{j i i}} \prod_{1 \leq i<j<k \leq r}\left[x_{j}, x_{i}, x_{k}\right]^{\gamma_{j i k}}\left[x_{k}, x_{i}, x_{j}\right]^{\gamma_{k i j}}$,

where $\gamma_{i}, \gamma_{j i k}$, and $\gamma_{k i j}$ are integers modulo $2^{\alpha_{i}} ; \gamma_{j i}$ is an integer modulo $2^{\alpha_{i}+1}$; $\gamma_{j i i}$ is an integer modulo $2^{\alpha_{i}-1}$; and $\gamma_{j i j}$ is an integer modulo $2^{\alpha_{i}-1}$ if $\alpha_{i}=\alpha_{j}$, and modulo $2^{\alpha_{i}}$ if $\alpha_{i}<\alpha_{j}$.

Struik also provides multiplication formulas in [19, pp. 453], which may be verified by applying the collection process; note however that our choice of basic commutators differs slightly from hers, so the formulas in [19] do not apply as is to our choice of normal forms. It is straightforward to do the necessary conversions. In any case we will not need the multiplication formulas here.

To simplify notation we set $\alpha=\alpha_{r-1}$. Let $G$ be as above; we want to determine the center of $G$. Clearly, $G_{3} \subset Z(G)$, and from Lemma 3.18 we know that $x_{r}^{2^{\alpha+1}}$ is central. By considering $G / G_{3}$, we obtain the 2-nilpotent product of the $C_{i}$, and so we know from Lemma 4.1 that the center of $G$ satisfies:

$$
\left\langle x_{r}^{2^{\alpha+1}}, G_{3}\right\rangle \subset Z(G) \subset\left\langle x_{r}^{2^{\alpha}}, G_{2}\right\rangle .
$$

We claim that in fact

$$
\left\langle x_{r}^{2^{\alpha+1}}, G_{3}\right\rangle \subset Z(G) \subset\left\langle x_{r}^{2^{\alpha+1}}, G_{2}\right\rangle .
$$

This can be seen as follows: if $\alpha=\alpha_{r}$, then $x_{r}^{2^{\alpha+1}}=x_{r}^{2^{\alpha}}=e$, and the claim is true. If, on the other hand, we have $\alpha<\alpha_{r}$, then consider the commutator of $x_{r-1}$ with $x_{r}^{2^{\alpha}}$. From (2.5) we have:

$$
\begin{aligned}
{\left[x_{r}^{2^{\alpha}}, x_{r-1}\right] } & =\left[x_{r}, x_{r-1}\right]^{2^{\alpha}}\left[x_{r}, x_{r-1}, x_{r}\right]^{\left(2_{2}^{\alpha}\right)} \\
& =\left[x_{r}, x_{r-1}\right]^{2^{\alpha}-2^{\alpha}}\left[x_{r}^{2}, x_{r-1}\right]^{2^{\alpha-1}} \\
& =\left[x_{r}^{2}, x_{r-1}\right]^{2^{\alpha-1}}
\end{aligned}
$$


where we have used the identity $\left[x_{j}, x_{i}, x_{j}\right]=\left[x_{j}, x_{i}\right]^{-2}\left[x_{j}^{2}, x_{i}\right]$, which may be obtained from (2.5) as well. Since $\alpha=\alpha_{r-1}<\alpha_{r}$, the order of $\left[x_{r}^{2}, x_{r-1}\right]$ is $2^{\alpha}$, so this commutator is not trivial. Therefore, $x_{r}^{2^{\alpha_{r-1}}}$ is not central, but its square is, giving once again the inclusions claimed.

Now let $g \in Z(G)$; multiplying by suitable powers of $x_{r}$ and of commutators of the form $\left[x_{j}, x_{i}, x_{k}\right]$ and $\left[x_{k}, x_{i}, x_{j}\right]$ we may assume that:

$$
g=\prod_{1 \leq i<j \leq r}\left[x_{j}, x_{i}\right]^{\gamma_{j i}}\left[x_{j}^{2}, x_{i}\right]^{\gamma_{j i j}}\left[x_{j}, x_{i}^{2}\right]^{\gamma_{j i i}} .
$$

We first take the commutator of $g$ with $x_{r}$; from (2.3) and Proposition 2.2(ii) we have:

$$
\begin{aligned}
{\left[g, x_{r}\right] } & =\prod_{1 \leq i<j \leq r}\left[x_{j}, x_{i}, x_{r}\right]^{\gamma_{j i}}\left[x_{j}^{2}, x_{i}, x_{r}\right]^{\gamma_{j i j}}\left[x_{j}, x_{i}^{2}, x_{r}\right]^{\gamma_{j i i}} \\
& =\prod_{1 \leq i<j \leq r}\left[x_{j}, x_{i}, x_{r}\right]^{\gamma_{j i}+2 \gamma_{j i j}+2 \gamma_{j i i}} .
\end{aligned}
$$

For $j<r$, we conclude that $\gamma_{j i}+2 \gamma_{j i j}+2 \gamma_{j i i} \equiv 0\left(\bmod 2^{\alpha_{i}}\right)$. If $j=r$ we again use the identity $\left[x_{r}, x_{i}, x_{r}\right]=\left[x_{r}, x_{i}\right]^{-2}\left[x_{r}^{2}, x_{i}\right]$ and conclude that we must have $-2\left(\gamma_{r i}+2 \gamma_{r i r}+2 \gamma_{r i i}\right) \equiv 0\left(\bmod 2^{\alpha_{i}+1}\right)$ by looking at the exponent of $\left[x_{r}, x_{i}\right]$ in the resulting expression. So in any case we conclude that

$$
\gamma_{j i}+2 \gamma_{j i j}+2 \gamma_{j i i} \equiv 0 \quad\left(\bmod 2^{\alpha_{i}}\right) ; \quad 1 \leq i<j \leq r .
$$

Conversely, it is easy to verify that if $g$ as above satisfies this condition, then it will necessarily be central in $G$. We obtain:

Theorem 5.1. Let $C_{1}, \ldots, C_{r}$ be cyclic groups, generated by $x_{1}, \ldots, x_{r}$ respectively; assume that the order of $x_{i}$ is $2^{\alpha_{i}}$, and that $1 \leq \alpha_{1} \leq \alpha_{2} \leq \cdots \leq \alpha_{r}$. Let $G$ be the 3-nilpotent product of the $C_{i}, G=C_{1} \amalg^{\mathfrak{N}_{3}} \cdots \amalg^{\mathfrak{N}_{3}} C_{r}$, and write $\alpha=\alpha_{r-1}$. Then $g \in Z(G)$ if and only if it can be written in normal form as:

$$
g=x_{r}^{\gamma_{r}} \prod_{1 \leq i<j \leq r}\left[x_{j}, x_{i}\right]^{\gamma_{j i}}\left[x_{j}, x_{i}^{2}\right]^{\gamma_{j i i}}\left[x_{j}^{2}, x_{i}\right]^{\gamma_{j i j}} \prod_{1 \leq i<j<k \leq r}\left[x_{j}, x_{i}, x_{k}\right]^{\gamma_{j i k}}\left[x_{k}, x_{i}, x_{j}\right]^{\gamma_{k i j}},
$$

where $\gamma_{r} \equiv 0\left(\bmod 2^{\alpha+1}\right)$, and $\rho_{j i} \equiv 0\left(\bmod 2^{\alpha_{i}}\right)$, where $\rho_{j i}=\gamma_{j i}+2 \gamma_{j i i}+2 \gamma_{j i j}$. That is

$$
Z(G)=\left\langle\left\{x_{r}^{2^{\alpha+1}}, G_{3}\right\} \bigcup\left\{\left[x_{j}, x_{i}\right]^{2^{\alpha_{i}}} \mid 1 \leq i<j \leq r\right\}\right\rangle .
$$

With a description of the center, we can now easily derive the characterization of the capable 2-nilpotent products of cyclic 2-groups:

Theorem 5.2. Let $C_{1}, \ldots, C_{r}$ be cyclic 2-groups generated by $x_{1}, \ldots, x_{r}$, respectively, where $2^{\alpha_{i}}$ is the order of $x_{i}$, and assume that $1 \leq \alpha_{1} \leq \cdots \leq \alpha_{r}$. If $G$ is the 2-nilpotent product of the $C_{i}$,

$$
G=C_{1} \amalg^{\mathfrak{N}_{2}} C_{2} \amalg^{\mathfrak{N}_{2}} \cdots \amalg^{\mathfrak{N}_{2}} C_{r},
$$

then $G$ is capable if and only if $r>1$ and $\alpha_{r} \leq \alpha_{r-1}+1$.

Proof. Necessity follows from Theorem 3.19 For sufficiency, let $K$ be the 3nilpotent product of the $C_{i}, K=C_{1} \amalg^{\mathfrak{N}_{3}} C_{2} \amalg^{\mathfrak{N}_{3}} \ldots \amalg^{\mathfrak{N}_{3}} C_{r}$. Then the description of the center at the end of Theorem 5.1 makes it easy to verify that $K / Z(K) \cong G$, so $G$ is capable. 
The preceding theorem suggests the following question:

Question 5.3. Is the k-nilpotent product of $r$ cyclic p-groups capable if and only if $r>1$ and

$$
\alpha_{r} \leq \alpha_{r-1}+\left\lfloor\frac{k-1}{p-1}\right\rfloor ?
$$

As we have seen, the answer is yes when $k<p$, and when $k=p=2$.

\section{Some APplications of OUR APPROACH}

As we noted in the introduction, one weakness of Theorems 4.5 and 5.2 is that whereas the 1-nilpotent product of cyclic groups covers all finitely generated abelian groups, the case $k \geq 2$ does not do the same for the finitely generated nilpotent groups of class $k$. However, it is possible to use our results as a starting point for discussing capability of other more general $p$-groups.

A recent result of Bacon and Kappe characterizes the capable 2-generated nilpotent $p$-groups of class two with $p$ an odd prime using the nonabelian tensor square [1. We can recover their result using our techniques, and easily obtain a bit more.

In Theorem 2.4 of 2 , the authors present a classification of the finite 2-generator $p$-groups of class two, $p$ an odd prime. With a view towards their calculations of the nonabelian tensor square, the authors classify the groups into three families. We will modify their classification and coalesce them into a single presentation.

Let $G=\langle a, b\rangle$ be a finite nonabelian 2-generator $p$-group of class $2, p$ an odd prime. Then $G$ is isomorphic to the group presented by:

$$
\left\langle\begin{array}{r|r}
a^{p^{\alpha}}=b^{p^{\beta}}=[b, a]^{p^{\gamma}} & =e, \\
{[a, b, a]=[a, b, b]} & =e, \\
a^{p^{\alpha+\sigma-\gamma}}[b, a]^{p^{\sigma}} & =e .
\end{array}\right\rangle
$$

where $\alpha+\sigma \geq 2 \gamma, \beta \geq \gamma \geq 1, \gamma \geq \sigma \geq 0$, and if $\sigma=\gamma$, then $\alpha \geq \beta$. Under these restrictions, the choice is uniquely determined.

If $\sigma=\gamma$, we obtain the groups in Bacon and Kappe's first family, which one might call the "coproduct type" groups (they are obtained from the nilpotent product $\langle a\rangle \amalg^{\mathfrak{N}_{2}}\langle b\rangle$ by taking the quotient modulo a power of $[a, b]$, and are the coproduct in a suitably chosen subvariety of $\mathfrak{N}_{2}$ ). If $\sigma=0$, we obtain the split meta-cyclic groups, which are the second family in [2]. The cases $0<\sigma<\gamma$ correspond to their third family.

The result which appears in [1] is that a 2-generated group with presentation as in 6.1 with $\sigma=0$ or $\sigma=\gamma$ is capable if and only if $\alpha=\beta$. The condition is also both necessary and sufficient for the remaining case with $0<\sigma<\gamma$ (in this case, 1 contains an error which the authors are in the process of correcting 13).

Thus, in the case of 2-generated $p$-groups of class two, $p$ an odd prime, Baer's condition is both necessary and sufficient, just as for finite abelian groups.

Although we could prove the result for all three families at once, we will divide the argument in two, to prove slightly more for the case where $\sigma=\gamma$.

Let $p$ be an odd prime and $C_{1}, \ldots, C_{r}$ be cyclic $p$-groups generated by $x_{1}, \ldots, x_{r}$, respectively, where $p^{\alpha_{i}}$ is the order of $x_{1}$ with $1 \leq \alpha_{1} \leq \alpha_{2} \leq \cdots \leq \alpha_{r}$. Let $K$ be the 2-nilpotent product of the $C_{i}$,

$$
K=C_{1} \amalg^{\mathfrak{N}_{2}} \ldots \amalg^{\mathfrak{N}_{2}} C_{r} .
$$


For each pair $j, i$, let $\beta_{j i}$ be a positive integer less than or equal to $\alpha_{i}$, and set $N=\left\langle\left[x_{j}, x_{i}\right]^{p^{\beta_{j i}}}\right\rangle$. Since $N$ is central, we have $N \triangleleft K$. Let $G=K / N$.

Theorem 6.2. The group $G$, as defined in the previous paragraph, is capable if and only if $r>1$ and $\alpha_{r-1}=\alpha_{r}$.

Proof. Necessity follows from Theorem 3.19 For sufficiency, let $H$ be the 3nilpotent product of the $C_{i}, H=C_{1} \amalg^{\mathfrak{N}_{3}} \cdots \amalg^{\mathfrak{N}_{3}} C_{r}$. Clearly $H$ will not do, so we need to take a quotient of $H$ so that, in the resulting group, $\left[x_{j}, x_{i}\right]^{\beta_{j i}}$ is central. To that end, we let $M$ be the subgroup of $H$ generated by all elements of the form $\left[x_{j}, x_{i}, x_{k}\right]^{\beta_{j i}}$ with $1 \leq i<j \leq r$, and $k$ arbitrary. In terms of the basic commutators, this means the elements $\left[x_{j}, x_{i}, x_{k}\right]^{\beta_{j i}}$ for $k \geq i$, and the elements

$$
\left[x_{i}, x_{k}, x_{j}\right]^{-\beta_{j i}}\left[x_{j}, x_{k}, x_{i}\right]^{\beta_{j i}}
$$

for $k<i$. It is easy to verify that all elements of $H / M$ have a normal form as in Theorem 3 of [19], and that the multiplication of these elements uses the same formulas as those in $H$, except that the exponents of the basic commutators of weight 3 are now taken modulo the adequate $\beta_{j i}$ instead of the old moduli.

Proceeding now as in the proof of Theorem 4.4 one proves that the center of $H / M$ is generated by the third term of the lower central series, the image of $x_{r}^{p^{\alpha_{r-1}}}$, and those of the elements $\left[x_{j}, x_{i}\right]^{p^{\beta_{j i}}}$; therefore by taking the central quotient, we obtain the group $G$, as desired.

The first part of [1, Corollary 4.3] corresponds to the case $r=2, x_{1}=a, x_{2}=b$, and $\beta_{21}=\gamma$. Now we consider the case with $\sigma<\gamma$.

Theorem 6.3 (cf. 11, Corollary 4.3]). Let $p$ be an odd prime, and let $G$ be a group presented by (6.1) with $0 \leq \sigma<\gamma$. Then $G$ is capable if and only if $\alpha=\beta$.

Proof. Necessity once again follows from Theorem 3.19 so we only need to prove sufficiency. The idea is to construct the "obvious witness" to the capability, by starting with the 3-nilpotent product of two cyclic groups of order $p^{\alpha}$, generated by $x$ and $y$; then for every relation $r$ in the presentation of $G$, we will take the quotient modulo the normal closure of $\langle[r, x],[r, y]\rangle$, thus making $r$ central in the quotient. Then we just need to make sure that in the resulting group, the map $x \mapsto a, y \mapsto b$ will yield the desired isomorphism between the central quotient and $G$. In essence, what we are doing is constructing a "generalized extension of $G$ " which can be used to determine the capability of $G$. See [4, Theorem III.3.9].

So let $H=\langle x\rangle \amalg^{\mathfrak{N}_{3}}\langle y\rangle$, where $x$ and $y$ are both of order $p^{\alpha}$.

First, we want to make sure that $[y, x]^{p^{\gamma}}$ is central. To that end, we let $N=$ $\left\langle[y, x, x]^{p^{\gamma}},[y, x, y]^{p^{\gamma}}\right\rangle$, and consider $K=H / N$.

The next step is to ensure that $x^{p^{\alpha+\sigma-\gamma}}[y, x]^{p^{\sigma}}$ is central. So first we consider

$$
\begin{aligned}
{\left[x^{p^{\alpha+\sigma-\gamma}}[y, x]^{p^{\sigma}}, x\right] } & =\left[x^{p^{\alpha+\sigma-\gamma}}, x\right]\left[x^{p^{\alpha+\sigma-\gamma}}, x,[y, x]^{p^{\sigma}}\right]\left[[y, x]^{p^{\sigma}}, x\right] \\
& =[y, x, x]^{p^{\sigma}}
\end{aligned}
$$

(using (2.3) and Proposition 2.2). So we let $L=K /\left\langle[y, x, x]^{p^{\sigma}}\right\rangle$.

So far, the only difference in the normal form and multiplication tables for $L$ and for $H$ is the order of $[y, x, x]$ and $[y, x, y]$. 
The final quotient we need to take is to ensure that $x^{p^{\alpha+\sigma-\gamma}}[y, x]^{p^{\sigma}}$ also commutes with $y$. To that end, we consider:

$$
\begin{aligned}
{\left[x^{p^{\alpha+\sigma-\gamma}}[y, x]^{p^{\sigma}}, y\right] } & =\left[x^{p^{\alpha+\sigma-\gamma}}, y\right]\left[x^{p^{\alpha+\sigma-\gamma}}, y,[y, x]^{p^{\sigma}}\right]\left[[y, x]^{p^{\sigma}}, y\right] \\
& =\left[x^{p^{\alpha+\sigma-\gamma}}, y\right][y, x, y]^{p^{\sigma}} \\
& =[y, x]^{-p^{\alpha+\sigma-\gamma}}[y, x, x]^{-\left(\begin{array}{c}
p^{\alpha+\sigma-\gamma} \\
2
\end{array}\right)}[y, x, y]^{p^{\sigma}} .
\end{aligned}
$$

The last equality uses (2.5). Note, however, that the conditions on $\alpha, \sigma$, and $\gamma$ imply that $\alpha+\sigma-\gamma>\sigma$, so we can simplify the expression above to:

$$
\left[x^{p^{\alpha+\sigma-\gamma}}[y, x]^{p^{\sigma}}, y\right]=[y, x]^{-p^{\alpha+\sigma-\gamma}}[y, x, y]^{p^{\sigma}}
$$

Let $N=\left\langle[y, x]^{p^{\alpha+\sigma-\gamma}}[y, x, y]^{-p^{\sigma}}\right\rangle$. It is easy to verify that for all $g \in L$, both $[g, x]$ and $[g, y]$ lie in $N$ if and only if

$$
g \in\left\langle x^{p^{\alpha+\sigma-\gamma}}[y, x]^{p^{\sigma}},[y, x]^{p^{\gamma}},[y, x, x],[y, x, y]\right\rangle,
$$

(for example, using the explicit multiplication formulas in [19], suitably modified to fit our choice of basic commutators) from which we deduce that if $M=L / N$, then $M / Z(M) \cong G$, as desired.

Let $p$ be an odd prime, and let $G$ be a noncyclic 2-generator finite $p$-group of class at most two. If $G=\langle a, b\rangle$, then at least one of $a$ and $b$ must have the same exponent as the exponent of the element of highest order of the group, since $G$ is regular; say $a$, with order $p^{\mu}$. Among all elements $b^{\prime} \in G$ such that $G=\left\langle a, b^{\prime}\right\rangle$, pick the one of smallest order and replace $b$ with $b^{\prime}$; say the order is $p^{\nu}$. Then $\mu$ and $\nu$ are invariants of $G$; in fact, they are the two largest type invariants of the group (see [12, §4]). Arguing as in 22 Lemma 2.3], one shows that $\langle a\rangle \cap\left\langle b^{\prime}\right\rangle=\{e\}$, and then following the argument in [2, Th. 2.4], it is easy to verify that $\mu$ and $\nu$ are equal to $\alpha$ and $\beta$ (in some order) in the presentation given in (6.1). So we obtain:

Corollary 6.4. Let $p$ be an odd prime, and let $G$ be a noncyclic 2-generator finite p-group of class at most two, and let $\alpha$ and $\beta$ be the two largest type invariants of $G$. Then $G$ is capable if and only if $\alpha=\beta$.

At this point, an obvious question to ask is whether the necessary condition of Theorem 3.19 will also prove to be necessary for the case of nilpotent groups of class two, as it was for abelian groups (at least, for odd prime and a suitable choice of minimal generating set). Unfortunately, the answer to that question is no.

Recall that a $p$-group $G$ is extra-special if and only if $G^{\prime}=Z(G),\left|G^{\prime}\right|=p$, and $G^{\mathrm{ab}}$ is of exponent $p$. The theorem of Beyl, Felgner, and Schmid in 5] mentioned in the introduction states that an extra-special $p$-group is capable if and only if it is dihedral of order order 8 , or of order $p^{3}$ and exponent $p$, with $p>2$. So the extraspecial $p$-group $G$ of order $p^{5}$ generated by $x_{1}, x_{2}, x_{3}, x_{4}$, and satisfying $\left[x_{3}, x_{1}\right]=$ $\left[x_{3}, x_{2}\right]=\left[x_{4}, x_{1}\right],\left[x_{4}, x_{2}\right]=\left[x_{4}, x_{3}\right]=\left[x_{2}, x_{1}\right]=e, x_{1}^{p}=x_{2}^{p}=x_{3}^{p}=x_{4}^{p}=e$, and $G_{2} \subset Z(G)$, is not capable, and is minimally generated by four elements of exponent $p$. Thus, the necessary condition is not sufficient in general for groups in $\mathfrak{N}_{2}$. An independent proof that $G$ is not capable together with some other applications using our methods for groups of exponent $p$ and class 2 appears in [15.

For now, we note that the nilpotent product can be used to produce a natural "candidate for witness" to the capability of a given finite nilpotent $p$-group $G$. Let $G$ be a finite nilpotent group of class $k>0$, minimally generated by elements 
$x_{1}, \ldots, x_{r}$. Let $w_{1}\left(x_{1}, \ldots, x_{r}\right), \ldots, w_{n}\left(x_{1}, \ldots, x_{r}\right)$ be words in $x_{1}, \ldots, x_{r}$ that give a presentation for $G$, that is:

$$
G=\left\langle x_{1}, \ldots, x_{r} \mid w_{1}\left(x_{1}, \ldots, x_{r}\right), \ldots, w_{n}\left(x_{1}, \ldots, x_{r}\right)\right\rangle .
$$

Let $\left\langle y_{1}\right\rangle, \ldots,\left\langle y_{r}\right\rangle$ be infinite cyclic groups, let $K=\left\langle y_{1}\right\rangle \amalg^{\mathfrak{N}_{k+1}} \cdots \amalg^{\mathfrak{N}_{k+1}}\left\langle y_{r}\right\rangle$, and let $N=\left\langle w_{1}\left(y_{1}, \ldots, y_{r}\right), \ldots, w_{n}\left(y_{1}, \ldots, y_{r}\right)\right\rangle^{K}$, that is, the normal closure of the subgroup generated by the words evaluated at $y_{1}, \ldots, y_{r}$. Finally, let $M=[N, K]$.

Theorem 6.5. The group $G$, as defined in the previous paragraph, is capable if and only if

$$
G \cong(K / M) / Z(K / M)
$$

where $K$ and $M$ are also as defined in the previous paragraph.

Proof. We only need to prove the "only if" part. Note that the map that sends $y_{1}, \ldots, y_{r}$ to $x_{1}, \ldots, x_{r}$ respectively gives a well-defined map from $K$ to $G$, and that $K /\left\langle N, K_{k+1}\right\rangle \cong G ;$ since $M \subseteq N$,

$$
(K / M) /\left\langle N M, K_{k+1} M\right\rangle \cong G,
$$

and $\left\langle N M, K_{k+1} M\right\rangle$ is central in $K / M$. Therefore, $(K / M) / Z(K / M)$ is a quotient of $G$. We want to show that it is actually isomorphic to $G$.

Since $G$ is capable, there is a group $H$ such that $H / Z(H) \cong G$. Let $h_{1}, \ldots, h_{r}$ be elements of $H$ that map to $x_{1}, \ldots, x_{r}$, respectively. Replacing $H$ by $\left\langle h_{1}, \ldots, h_{r}\right\rangle$ if necessary, we may assume that $h_{1}, \ldots, h_{r}$ generate $H$. Since $G$ is of class $k$, $H$ is of class $k+1$, and therefore there exists a unique surjective map from $K$ (the relatively free $\mathfrak{N}_{k+1}$ group of rank $r$ ) to $H$, mapping $y_{1}, \ldots, y_{r}$ to $h_{1}, \ldots, h_{r}$, respectively. We must have that $w_{i}\left(h_{1}, \ldots, h_{r}\right) \in Z(H)$ for $i=1, \ldots, n$, so the map from $K$ to $H$ factors through $K / M$. Since $Z(K / M)$ maps into $Z(H)$, the induced mapping $K / M \rightarrow H \rightarrow G$ factors through $(K / M) / Z(K / M)$. Therefore, we have that $(K / M) / Z(K / M)$ has $G$ as a quotient. Since it is also isomorphic to a quotient of the finite group $G$, we must have $(K / M) / Z(K / M) \cong G$, as claimed.

\section{ACKNOWLEDGEMENTS}

I became interested in the question of capability after attending talks by L.C. Kappe and M. Bacon at the AMS Meeting in Baltimore. They were kind enough to give me a copy of their preprint of [1], and L.C. Kappe very patiently answered my questions over e-mail. I thank them both very much for their time and help.

In addition, several people were kind enough to help me during the preparation of this paper. Bill Dubuque, Ed Hook, Robert Israel, and Joseph Silverman helped with some of the calculations relating to binomial coefficients. George Bergman offered helpful comments. Avinoam Mann brought several known results to my attention. Derek Holt, Mike Newman, and Vasily Bludov from the Group Pub Forum verified some of my hand-calculations using MAGMA. Finally, Robert Morse used GAP to verify some of my constructions, answered many of my questions, and also provided some counterexamples to guesses of mine. I thank all of them very much for their time and their help.

Finally, I am very grateful to the referee, who offered many suggestions that greatly improved readability, as well as pointing out a gap in the original proof of Lemma 3.14 and suggesting the statement of Corollary 6.4 
Errata. Added 4/10/2006. Updated 5/15/2006.

The last clause of Lemma 4.2(ii) as stated above is unfortunately false. For example, if the weight of $\left[v, x_{r}\right]$ is smaller than the weight of $u$, then $\left[v, x_{r}, u\right]$ cannot be a basic commutator; in addition, if $u$ is of the form $\left[c_{1}, c_{2}\right]$ with $c_{i}$ a basic commutator and the weight of $c_{2}$ is greater than 1 , then $\left[u, x_{r}\right]$ is not a basic commutators, hence neither is $\left[u, x_{r}, v\right]$. Analogous problems arise if $v$ is of this form.

The problem can be resolved by continuing the process, using work of Ward and of T.C. Hurley; a proof of Theorem 4.4 follows from those arguments. I am preparing a second part which will contain the correction of the problem, as well as extending the results to $k=p$ for arbitrary prime $p$.

\section{REFERENCES}

[1] Michael R. Bacon and Luise-Charlotte Kappe, On capable p-groups of nilpotency class two, Illinois J. Math. 47 (2003), 49-62.

[2] - The nonabelian tensor square of a 2-generator p-group of class 2, Arch. Math. (Basel) 61 (1993), 508-516. MR 95h:20041

[3] Reinhold Baer, Groups with preassigned central and central quotient group, Trans. Amer. Math. Soc. 44 (1938), 387-412.

[4] F. Rudolf Beyl and Jürgen Tappe, Group extensions, representations, and the Schur multiplicator, Lecture Notes in Math., vol. 958, Springer-Verlag, 1982. MR 84f:20002

[5] F. Rudolf Beyl, Ulrich Felgner, and Peter Schmid, On groups occurring as central factor groups, J. Algebra 61 (1979), 161-177. MR 81i:20034

[6] Graham Ellis, On the capability of groups, Proc. Edinb. Math. Soc. (1998), 487-495. MR 2000e: 20053

[7] O. N. Golovin, Metabelian products of groups, Amer. Math. Soc. Transl. Ser. 22 (1956), 117-131. MR 17:824b

[8] _ Nilpotent products of groups, Amer. Math. Soc. Transl. Ser. 2 (1956), 89-115. MR 17:824a

[9] M. Hall, The theory of groups, Mac Millan Company, 1959. MR 21:1996

[10] M. Hall and J.K. Senior, The groups of order $2^{n}(n \leq 6)$, MacMillan and Company, 1964. MR 29: $\# 5889$

[11] P. Hall, The classification of prime-power groups, J. Reine Angew. Math. 182 (1940), 130141. MR 2,211b

[12] _ A contribution to the theory of groups of prime-power order, Proc. London Math. Soc. 36 (1934), 29-95.

[13] Luise-Charlotte Kappe, personal communication.

[14] T. MacHenry, The tensor product and the 2nd nilpotent product of groups, Math. Z. 73 (1960), 134-145. MR 22:11027a

[15] Arturo Magidin, Capable groups of prime exponent and class two, arXiv:math.GR/0401423.

[16] Dominions in varieties of nilpotent groups, Comm. Algebra 28 (2000), 1241-1270. MR 2000m:20053

[17] On the orders of generators of capable p-groups, arXiv:math.GR/0405087, to appear in Bull. Austral. Math. Soc.

[18] Hanna Neumann, Varieties of groups, Ergeb. Math. Grenzgeb. New Series, vol. 37, Springer Verlag, 1967. MR 35:6734

[19] Ruth Rebekka Struik, On nilpotent products of cyclic groups, Canad. J. Math. 12 (1960), 447-462. MR 22:11028

[20] - On nilpotent products of cyclic groups II, Canad. J. Math. 13 (1961), 557-568. MR 26:2486 